\title{
Visual field defects in obstructive hydrocephalus
}

\author{
PRD HUMPHREY, IF MOSELEY, RW ROSS RUSSELL \\ From the National Hospital for Nervous Diseases, Queen Square, London UK
}

SUMMARY Four cases are described in which visual field defects followed enlargement of the third ventricle. Three were due to aqueduct stenosis while in one case a left cerebellar hemisphere tumour was discovered. The visual field defects comprised a unilateral scotoma, bilateral scotomata and, in two patients, incongruous bitemporal hemianopia.

When the CSF pathway is chronically obstructed at the aqueduct or in the posterior fossa the resultant enlargement of the third ventricle is not uniform and bulging occurs where there is the least amount of brain tissue overlying the ventricular cavity, as at the lamina terminalis and in the suprapineal recess. Posterior herniation at the suprapineal site may extend backwards into the posterior fossa compressing the tectum of the mid brain and leading to pupillary and oculomotor changes. The effects of forward and downward expansion are also striking, although less commonly seen since effective ventricular shunting procedures have been introduced. Visual loss from distortion of the visual pathways by the third ventricle is a recognised feature of slowly growing posterior fossa tumours and of aqueduct stenosis but because of the coexistence of raised intracranial pressure it is difficult to separate the effects of local compression of the nerves or chiasm from those of chronic papilloedema of the optic nerve head.

We describe four patients in whom the dilated third ventricle produced compressive field defects at different points in the visual pathway. There was no evidence of previous papilloedema and only one patient had slight disc swelling at the time of presentation.

\section{Case 1}

A 9-year-old girl presented with a two months history of deteriorating reading and writing. Central vision was blurred in both eyes; this had begun suddenly one morning and tended to vary from day to day. She complained of headaches and mild unsteadiness for a few weeks. Ten months prior to presentation she had a severe bout of

Address for reprint requests: Dr PRD Humphrey, National Hospital for Nervous Diseases, Queen Square, London WC1 3BG, UK.

Received 4 December 1981 and in revised form 5 March 1982. Accepted 10 March 1982. measles. On examination, visual acuity was reduced to counting fingers in both eyes. Pupils were dilated and reacted sluggishly to direct light. Fundoscopy showed optic atrophy on the right with temporal pallor on the left. Visual field examination demonstrated bilateral central scotomata (fig 1). Skull radiographs showed a " $J$ " configuration sella turcica, with a short dorsum, marked suture separation and a large, thin vault (fig 2). Computed tomography $(\mathrm{CT})$ revealed aqueduct stenosis with a large, central third ventricle going down into the sella (fig 3). This was confirmed on ventriculography. Following a ventriculoperitoneal shunt her headaches and unsteadiness improved but there was no change in her vision.

\section{Case 2}

A 30-year-old man presented with blindness in the left eye. Visual acuity was $6 / 9$ on the right and counting fingers at two feet on the left. The left pupil was large and reacted sluggishly to direct light; the left disc was pale. A central scotoma was present on the left, the right visual field being normal (fig 4). Skull radiographs showed a " J" sella turcica with a truncated dorsum. CT demonstrated marked enlargement of the third and lateral ventricles suggestive of aqueduct stenosis. The appearances on the ventriculogram were consistent with an enlarged third ventricle, occupying most of the sella (fig 5). Torkildsen's procedure did not result in any significant change in visual acuity.

\section{Case 3}

A 12-year-old boy presented with a three month history of difficulty with reading and writing. He had also noticed difficulty in receiving a ball from the right. More recently he had had headaches associated with nausea suggestive of raised intracranial pressure. He was found to be small, his weight being on the 10 th centile and his height between the 3 rd and 10 th centile. Vision was reduced to $6 / 60$ on the right and $6 / 9$ on the left. Horizontal nystagmus was present on right lateral gaze. The pupils were equal in size but the right reacted sluggishly to direct light. Fundoscopy showed bilateral optic pallor with slight swelling of the disc margins. Visual field examination showed temporal loss with spread to central vision on the right (fig 6). Skull radiographs demonstrated an expanded posterior fossa. The sella turcica was " $\mathrm{J}$ " shaped without gross shortening of 


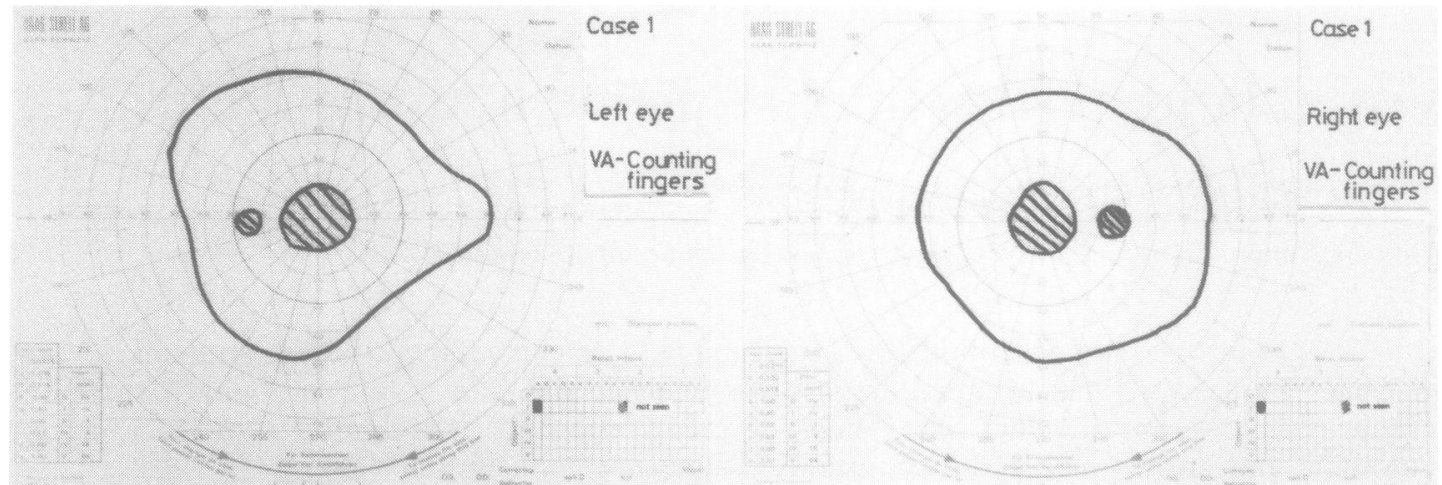

Fig 1 Case 1: visual fields showing bilateral central scotomata.

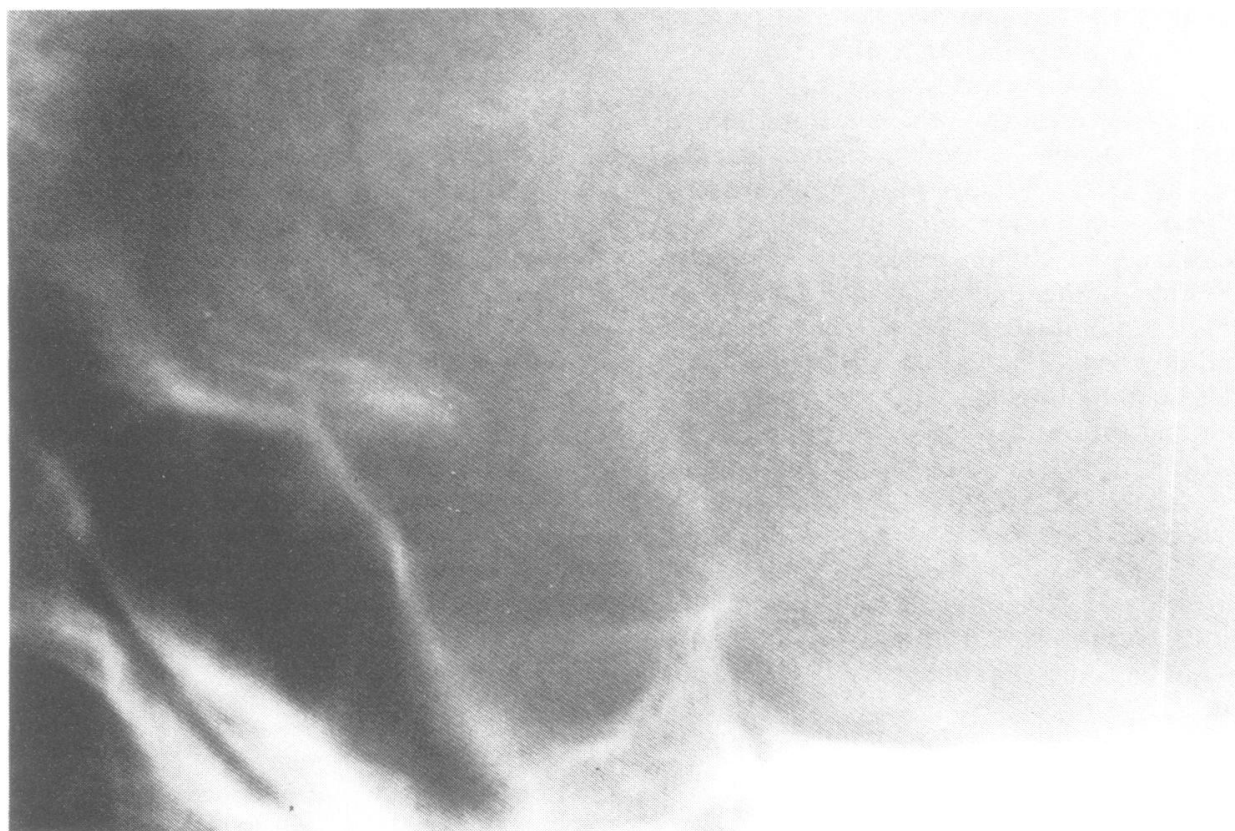

Fig 2 Case 1: skull radiograph: Typical "J" sella, with truncated dorsum, deep sulcus chiasmaticus and prominent anterior clinoid processes.

the dorsum sellae and the sutures were noted to be separated. CT showed the third ventricle to be $15 \mathrm{~mm}$ wide, symmetrically dilated and extending down into the sella. The lateral ventricles were also enlarged and a low attenuation lesion showing enhancement with intravenous contrast medium was present in the left cerebellar hemisphere. Following excision of an astrocytoma, there was no significant change in vision.

\section{Case 4}

A 22-year-old woman presented with a history of headache, diplopia and episodes of blurred vision. On examination, visual acuity was $6 / 9$ on the right and $6 / 18$ on the left and there was bilateral optic atrophy. Charting the visual fields demonstrated a temporal hemianopia in the left eye with temporal field loss on the right (fig 7). Skull radiographs showed a deep " J" shaped sella. CT showed hugely dilated lateral and third ventricles with the latter extending down into the sella region (fig 8). Ventriculography confirmed that the third ventricle was occupying almost the whole of the sella turcica (fig 9). A ventriculoatrial shunt was followed by a slight improvement in vision to $6 / 6$ on the right and $6 / 12$ on the left. 

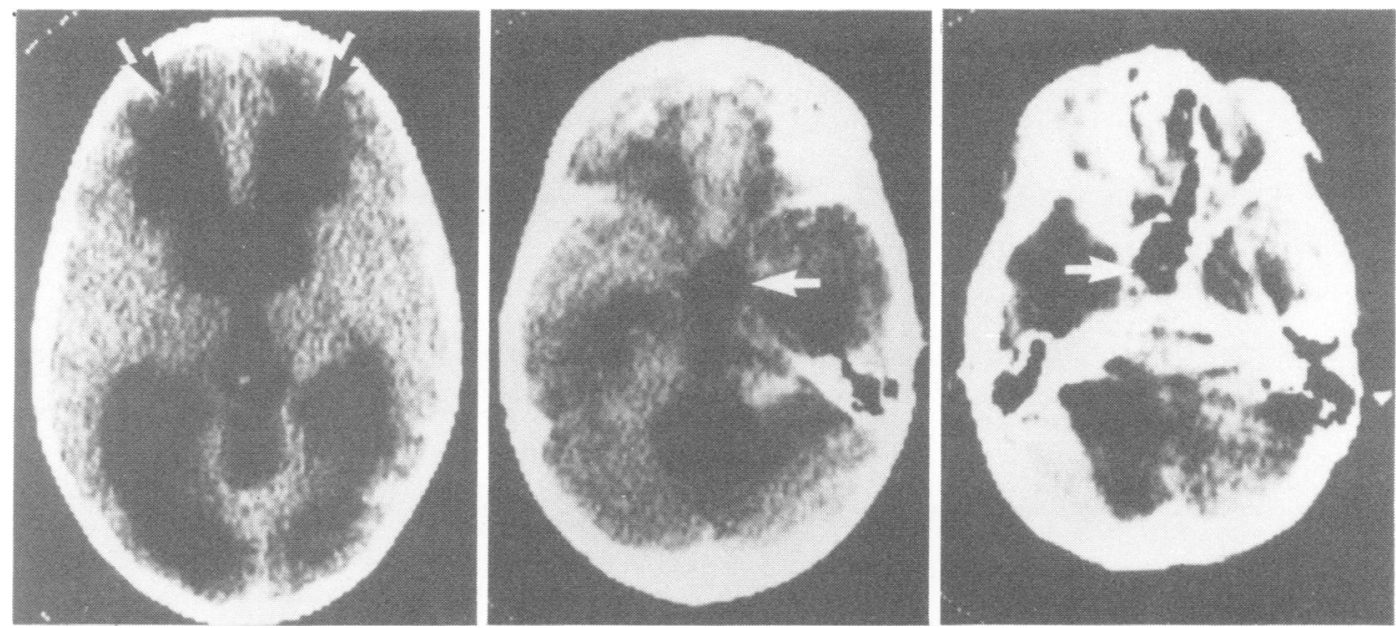

Fig 3 Case 1: CT: there is marked hydrocephalus involving all four ventricles; periventricular lucencies are present, especially around the frontal horns (black and white arrows). The dilated third ventricle extends right down to the sella region (horizontal arrows).

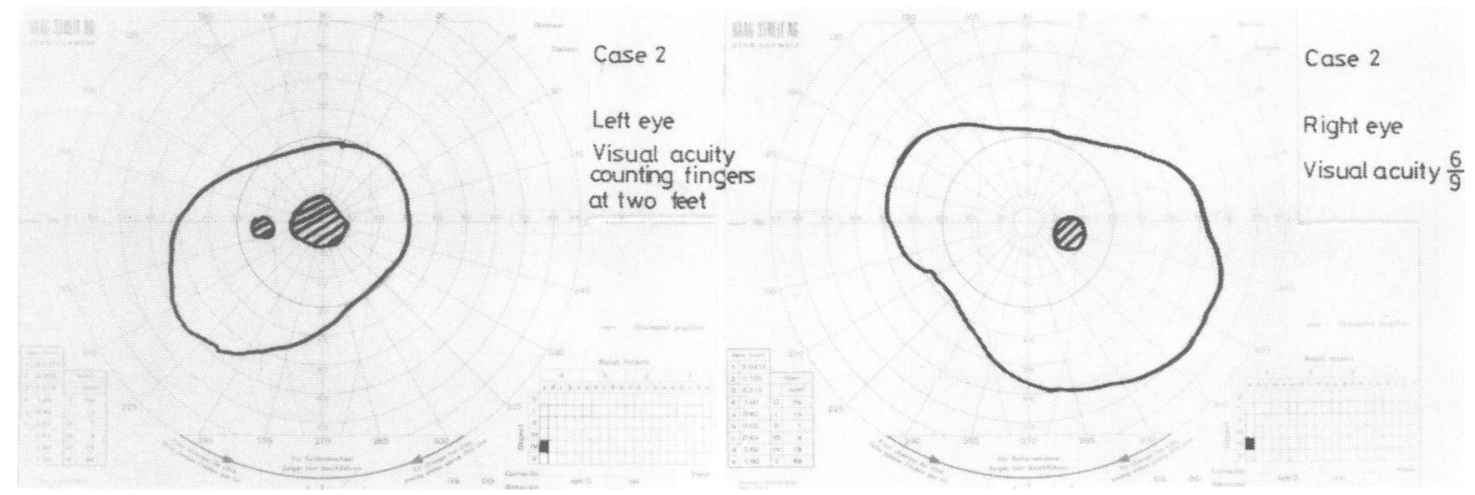

Fig 4 Case 2: visual fields showing left central scotoma.

\section{Discussion}

The pathogenesis of visual field defects in patients with posterior fossa lesions or aqueduct stenosis has been debated since the last century. Cushing and Walker were amongst the first to realise the importance of the dilated third ventricle acting as a mechanical compressive and distorting force on the visual pathways. ${ }^{1}$ They found binasal defects to be the most frequent result and proposed that the dilated ventricle displaced both optic nerves outwards against the resistant carotid arteries. The field defects shown by their patients indicate a generalised contraction of the fields more marked on the nasal side, which might well have been the result of chronic papilloedema. ${ }^{2}$ They encountered no patients with bitemporal hemianopia. However, bitemporal defects were convincingly shown by Sinclair and Dott in a patient with postmeningitic obstructive hydrocephalus. ${ }^{3}$ At operation the chiasm was shown to be broadened, and pushed downwards and forwards by the bulging anterior wall of the third ventricle. After ventriculostomy the vision improved. Hughes, in a careful study of the evolution of the field defect in third ventricular enlargement, stressed the importance of the position of the chiasm. ${ }^{4} \mathrm{He}$ suggested that if the chiasm was postfixed or in its normal position the enlarging third ventricle would impinge against the posterior aspect and spread over the superior surface tending to produce indrawing of the outer visual isopters of the temporal fields (depressional field). If on the other hand the chiasm was prefixed the ventricle pressed against its posterior and inferior aspects sometimes 


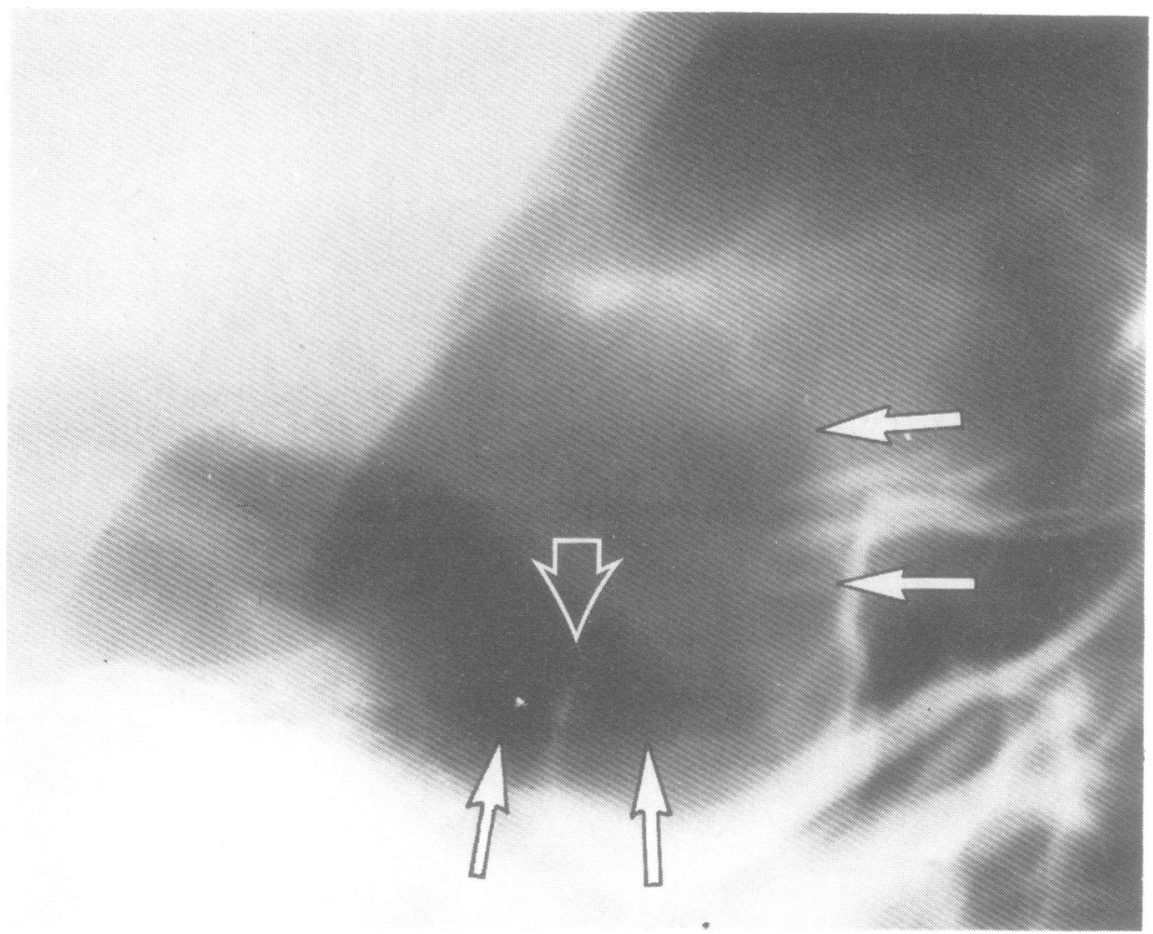

Fig 5 Case 2: ventriculogram, lateral projection: the anterior end of the third ventricle, grossly dilated (arrows), occupies the suprasella region, and much of the sella turcica, resting on the dorsum (open arrow).
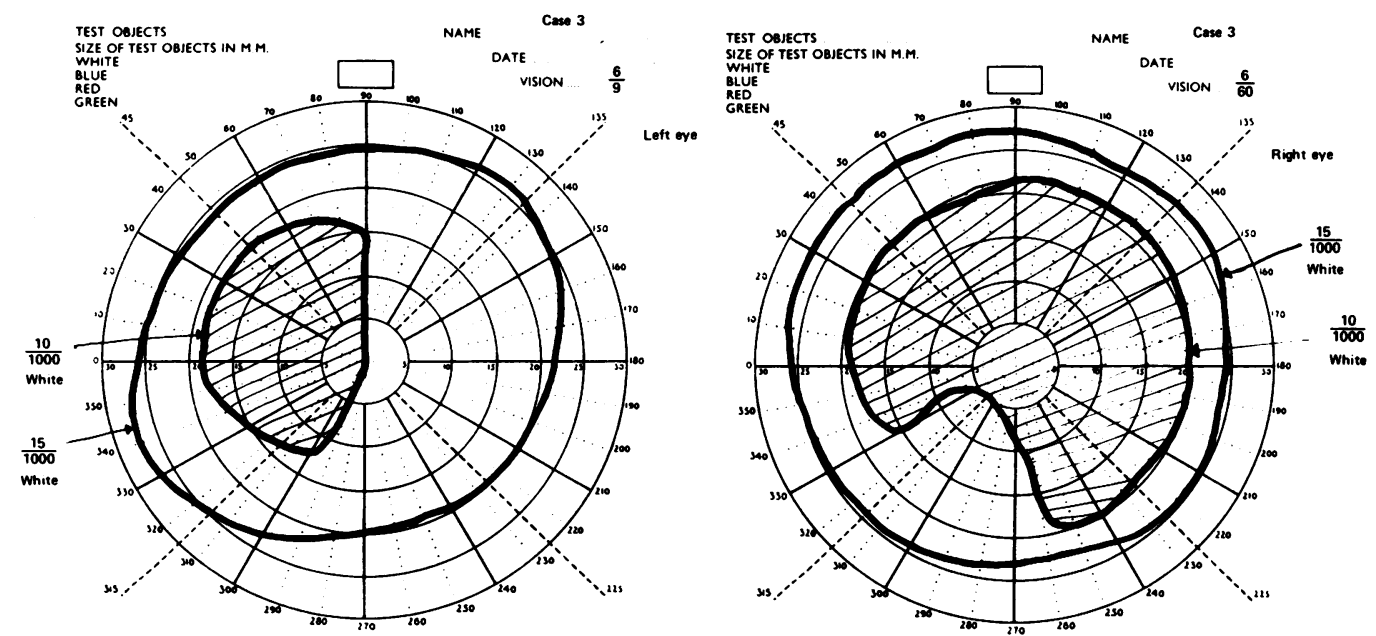

Fig 6 Case 3: bitemporal hemianopia with loss of central vision on the right. 

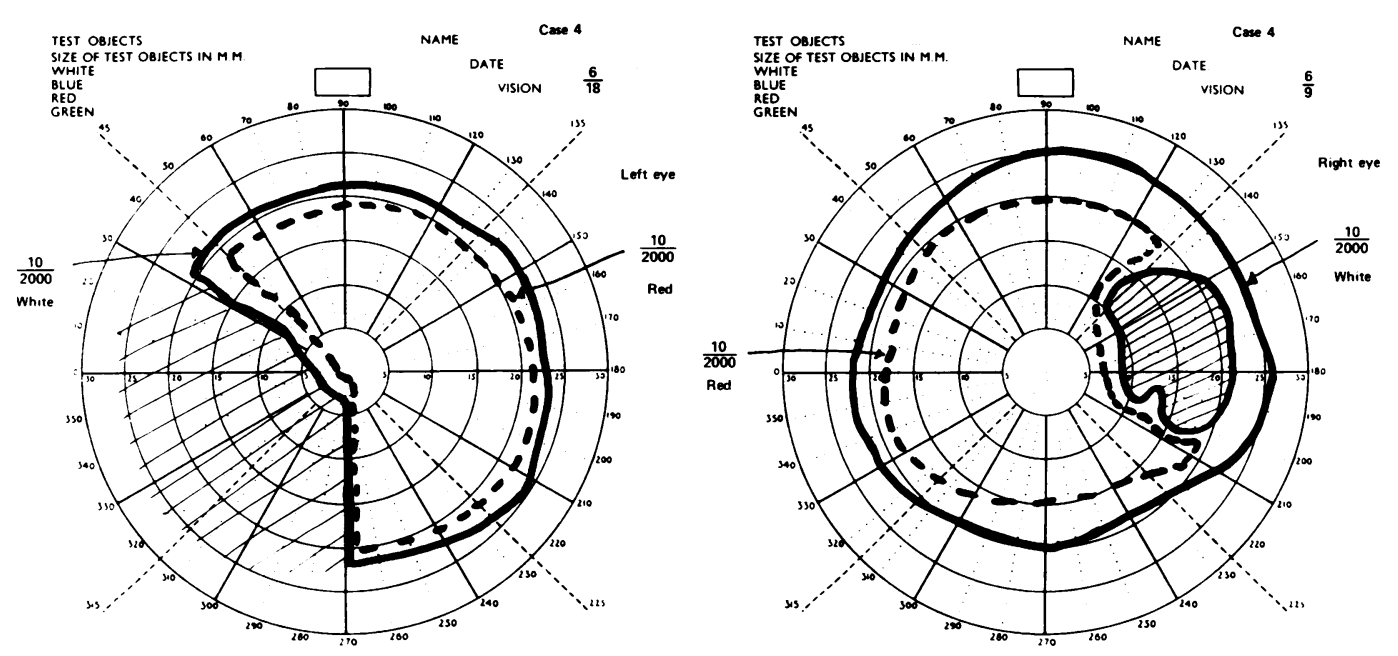

Fig 7 Case 4: visual fields demonstrating incongruous bitemporal hemianopia.

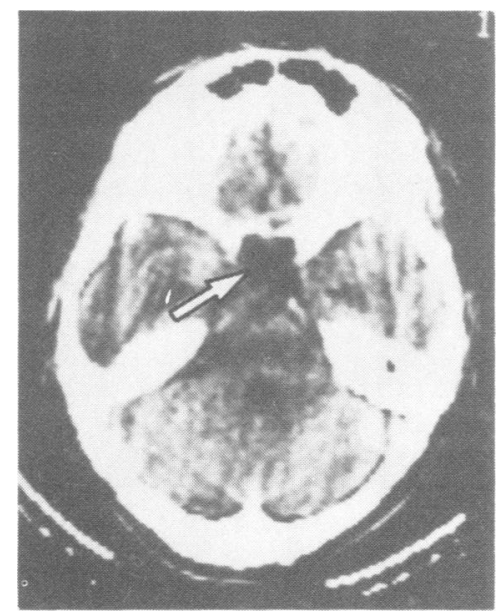

Fig 8 Case 4: CT: a widened anterior third ventricle (arrow) lies between the anterior clinoid processes, in the position normally occupied by the pituitary. The dorsum sellae is not seen. The fourth ventricle is normal. 


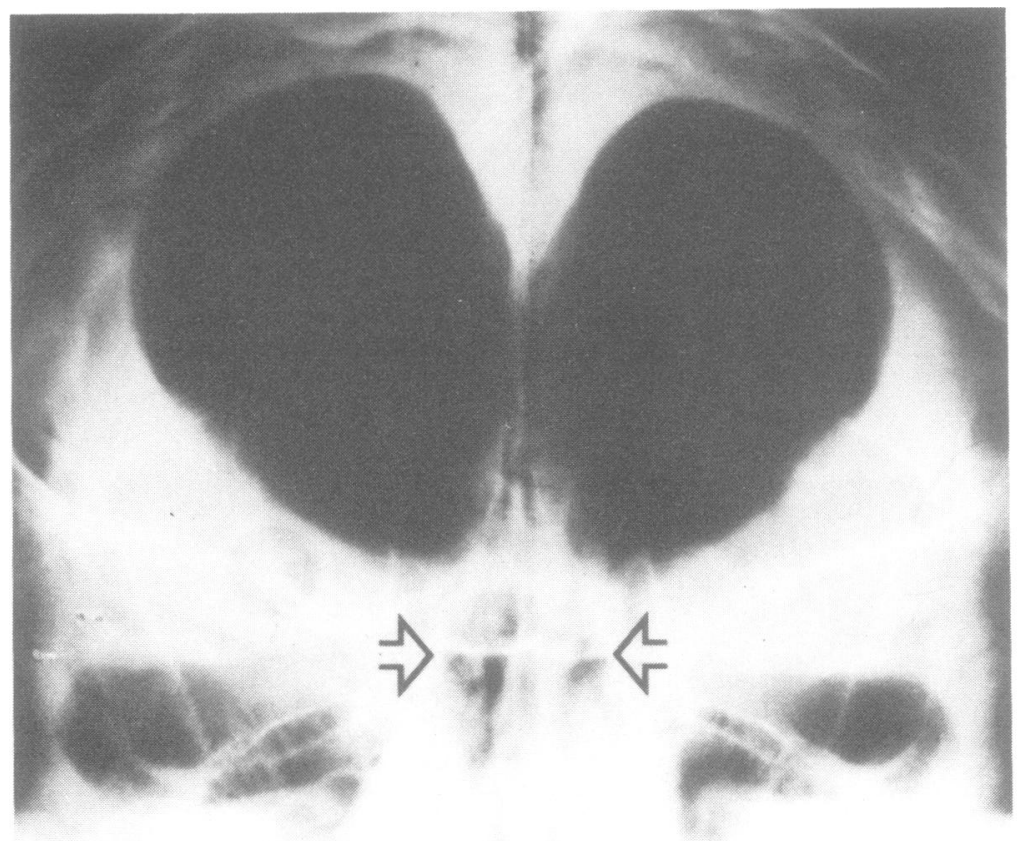

Fig 9 Case 4: ventriculogram. (a) anteroposterior, brow up projection. The lateral ventricles are grossly dilated, as is the third ventricle (open arrows). (b) lateral, brow up projection. An enlarged pituitary fossa is almost entirely occupied by the anterior end of the third ventricle (arrows), which has eroded the dorsum sellae (open arrow). The dense black central shadow is caused by air in both temporal horns superimposed on the third ventricle. 
bulging beneath it to lie between the optic nerves. In this case he proposed that the early field defects were bitemporal scotomas appearing first in the lower temporal quadrants in the paracentral region.

Lassman, Cullen and Howat, describing a patient with an upper temporal defect in one eye only, indicating a lesion near the junction of nerve and chiasm, proposed that the lower aspect of the nerve and chiasm were displaced downwards by the dilated ventricle and were pressed forcibly against the planum sphenoidale. ${ }^{5}$ Junctional defects of this type are also mentioned by Wagener and Cusick. ${ }^{6}$

Optic nerve defects are distinctly unusual. Bilateral central scotomas are mentioned by Wagener and Cusick in a catalogue of defects in patients with posterior fossa lesions and a single case was remarked upon by Pennybacker. ${ }^{7}$ A patient with complete unilateral blindness and a normal contralateral field was described by Calogero and Alexander. ${ }^{8}$ There has been some speculation as to how a central herniation could cause a unilateral optic nerve lesion but the close proximity of the anterior cerebral arteries, which are frequently unequal in size may result in compression of a distorted optic nerve.

Homonymous hemianopias have also been reported to result from an enlarged third ventricle. In the four patients with cerebellar tumours described by Weinberger and Webster ${ }^{9}$ the defects were congruous and might possibly have been the result of a posterior cerebral artery occlusion; the mechanism suggested by the authors was downward pressure on the tract by dilated third ventricle against the posterior communicating artery.

The present report is a further illustration of the variety of field defects. Two of our patients had bitemporal hemianopias (both asymmetrical), one a unilateral scotoma and one bilateral central scotomas. The latter defect is particularly unusual and is a possible source of misdiagnosis because while a progressive unilateral optic nerve lesion or bitemporal hemianopia always suggests a compressive lesion, a patient with bilateral central scotomas is more likely to have a demyelinating or toxic neuropathy than a compressive lesion.

No firm conclusions can be reached on the mechanism of visual loss. While enlargement of the third ventricle into the pituitary fossa is likely to be an important factor in the development of optic nerve, chiasmal or tract lesions, it is doubtful if this is the sole reason for these visual field defects. Schechter and Zingesser showed that in the ventriculogram in aqueduct stenosis the third ventricle frequently enlarges to occupy the pituitary fossa. ${ }^{10}$ Visual defects such as those reported here are, however, rare. The ventriculographic and CT appearances in our patients were typical of those found in patients with obstructive hydrocephalus. All four patients showed extension of the hypothalamic recess into the pituitary fossa rather than extension of the supraoptic recess over the chiasm. Enlargement of this type would be likely to impinge on the posterior aspect of the chiasm and since the chiasm bears a fixed relationship to the recesses it seems unlikely that pre or postfixation of the chiasm would assert a major influence on the type of field defect.

We are grateful to Mr J Garfield from the Wessex Neurological Centre for allowing us to report case 4.

\section{References}

${ }^{1}$ Cushing $\mathrm{H}$, Walker CB. Distortion of the visual fields in cases of brain tumour. Arch Ophthalmol 1912;41:559-98.

${ }^{2}$ Osher RH, Corbett JJ, Schatz NJ, Saisho PJ, Orr LS. Neuro-ophthalmological complications of enlargement of the third ventricle. Br J Ophthalmol 1978;62:536-42.

${ }^{3}$ Sinclair AHH, Dott NM. Hydrocephalus simulating tumour in the production of chiasmal and other parahypophysial lesions. Trans Ophthalmol Soc UK 1931;51:232-46.

${ }^{4}$ Hughes EBC. Some observations on the visual fields in hydrocephalus. J Neuro Neurosurg Psychiatry 1946;9:30-39.

${ }^{5}$ Lassman LP, Cullen JF, Howat JM. Stenosis of the aqueduct of Sylvius. Am J Ophthalmol 1960;49:261-6.

- Wagener HP, Cusick PL. Chiasmal syndromes produced by lesions in the posterior fossa. Arch Ophthalmol 1937;18:887-91.

' Pennybacker J. Stenosis of the aqueduct of Sylvius. Proc Soc Med 1940;33:507-12.

- Calogero JA, Alexander E. Unilateral amaurosis in a hydrocephalic child with an obstructed shunt. J Neurosurg 1971;34:236-40.

9 Weinberger LM, Webster JE. Visual field defects associated with cerebellar tumours. Arch Ophthalmol 1941;25:128-38.

${ }^{10}$ Schechter MM, Zingesser LH. The radiology of aqueductal stenosis. Radiology, 1967;88:905-16. 